\title{
Pulmonary vein isolation with rotational angiography based three-dimensional left atrial reconstruction
}

\author{
Bartosz Krzowski ${ }^{1, \mathrm{~B}, \mathrm{D}}$, Michal Peller ${ }^{1, \mathrm{~B}-\mathrm{D}}$, Paweł Balsam ${ }^{1, \mathrm{E}}$, Piotr Lodziński ${ }^{1, \mathrm{E}}$, Marcin Grabowski ${ }^{1, \mathrm{~A}, \mathrm{E}-\mathrm{F}}$, Grzegorz Opolski ${ }^{1, \mathrm{~F}}$ \\ A - Research concept and design, B - Collection and/or assembly of data, C - Data analysis and interpretation, \\ D - Writing the article, E - Critical revision of the article, F - Final approval of article
}

1 Medical University of Warsaw

Address for correspondence:

Bartosz Krzowski, Medical University of Warsaw email: bartekkrzowski@gmail.com

Michal Peller, Medical University of Warsaw email: michalpeller@gmail.com

Paweł Balsam, Medical University of Warsaw email: pawel.balsam@me.com

Piotr Lodziński, Medical University of Warsaw email: plodz@me.com

Marcin Grabowski, Medical University of Warsaw email: grabowski.marcin@me.com

Grzegorz Opolski, Medical University of Warsaw email: grzegorz.opolski@wum.edu.pl

Received: 2019-04-21

Revised: 2019-07-12

Accepted: 2019-07-12

Final review: 2019-06-16

DOI: $10.24255 / \mathrm{hbj} / 110684$

\section{Key words:}

atrial fibrillation, catheter ablation, Rotational angiography

\section{Abstract}

Atrial fibrillation remains the cardiac arrhythmia of highest relevance, exerting a huge burden on both societies and individuals. Together with constant technological progress, new methods are continually implemented in everyday practice, which translates into better prognosis and outcomes of invasive treatment. A crucial part of the pulmonary vein isolation procedure is proper reconstruction of cardiac structures. One

\section{Introduction}

Since atrial fibrillation (AF) is the most common supraventricular arrhythmia, with very dangerous complications, including stroke. There are several treatment protocols. Catheter ablation (CA) stands as the method of first choice when it comes to treatment of symptomatic atrial fibrillation ${ }^{(1)}$. In order to provide proper and safe pulmonary vein isolation (PVI) accurate visualization of the left atrium is required, which is highly demanding. Fluoroscopy and electro-anatomical maps (EAM) have been used for years with possible previous magnetic resonance imaging (MRI) or computed tomography (CT). However, only 3D rotational angiography (3DRA) in combination with electro-anatomical maps offers real-time high-quality imaging, and therefore is regarded as the gold standard when it comes to left atrium reconstruction for $\mathrm{CA}^{(2)}$. of the methods offering the highest vision quality is a combination of rotational angiography with electro-anatomical mapping. We present a case of a 62-year-old patient referred to our department diagnosed with paroxysmal atrial fibrillation and successfully treated with catheter ablation using visualization consisting of electro-anatomical mapping and rotational angiography.

We present the case of a patient with paroxysmal $\mathrm{AF}$ scheduled for CA, which was carried out using rotational angiography.

\section{Case report}

A 62-year-old patient was admitted to our department to undergo PVI, because of moderately symptomatic (EHRA $2 b)$ paroxysmal AF diagnosed 10 years before. The patient suffered from AF episodes approximately twice a year and required multiple electrical cardioversions in order to restore sinus rhythm. Moreover, the patient had a history of benign prostate hyperplasia and meningioma of the right parietal region. The patient was treated with dabigatran, tamsulosin and rosuvastatin.

Preprocedural echocardiography revealed ejection fraction $60 \%$ and left atrium (LA) size of $3.7 \mathrm{~cm}$. Transesophageal 
echocardiography ruled out thrombus presence in the left atrium appendage.

At the beginning of the procedure sinus rhythm was observed. Under the control of blood pressure values double transseptal puncture was performed (Figure 1). Both transsep-

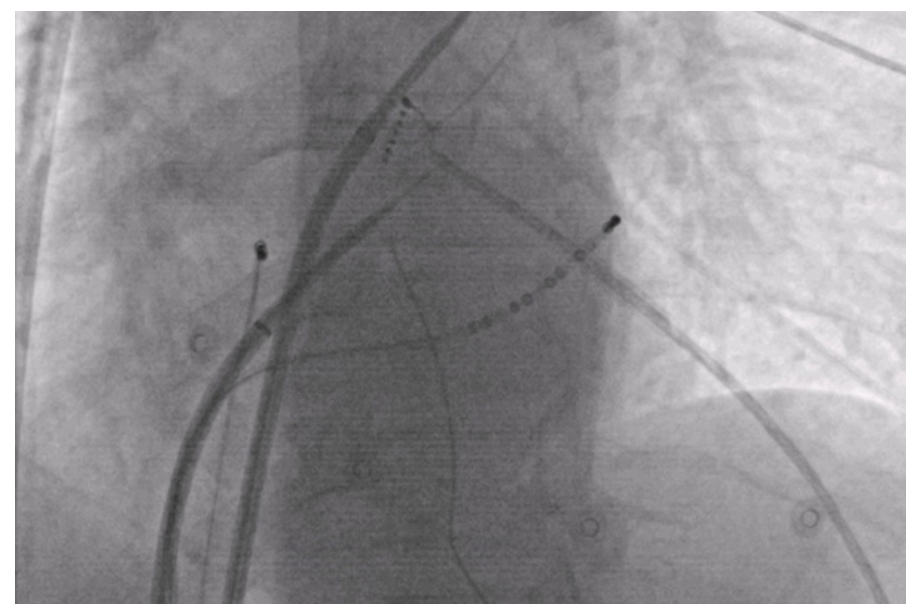

Figure1. Fluoroscopic view during catheter ablation procedure, LAO $30^{\circ}$ view (transseptal puncture).

tal sheaths with catheters LassoNav (20 points) and NaviSmart SF were entered into the left atrium. The transseptal sheath was rotated to the posterior wall of the LA. Rotation angiography along a circular arc was performed within $5 \mathrm{~s}$ with breath hold by the patient, according to the left atrial protocol. The patient was placed in a supine position with his arms in a natural position along the body. Contrast was injected by automatic pump, synchronized with the $X$-ray system, during rapid stimulation of the ventricles $(230 / \mathrm{min})$ in order to reduce cardiac output. Volume of contrast diluted 6:4 with saline was $100 \mathrm{~mL}(20 \mathrm{~mL} / \mathrm{s})$ (Figure 2,3). The type of X-ray system and

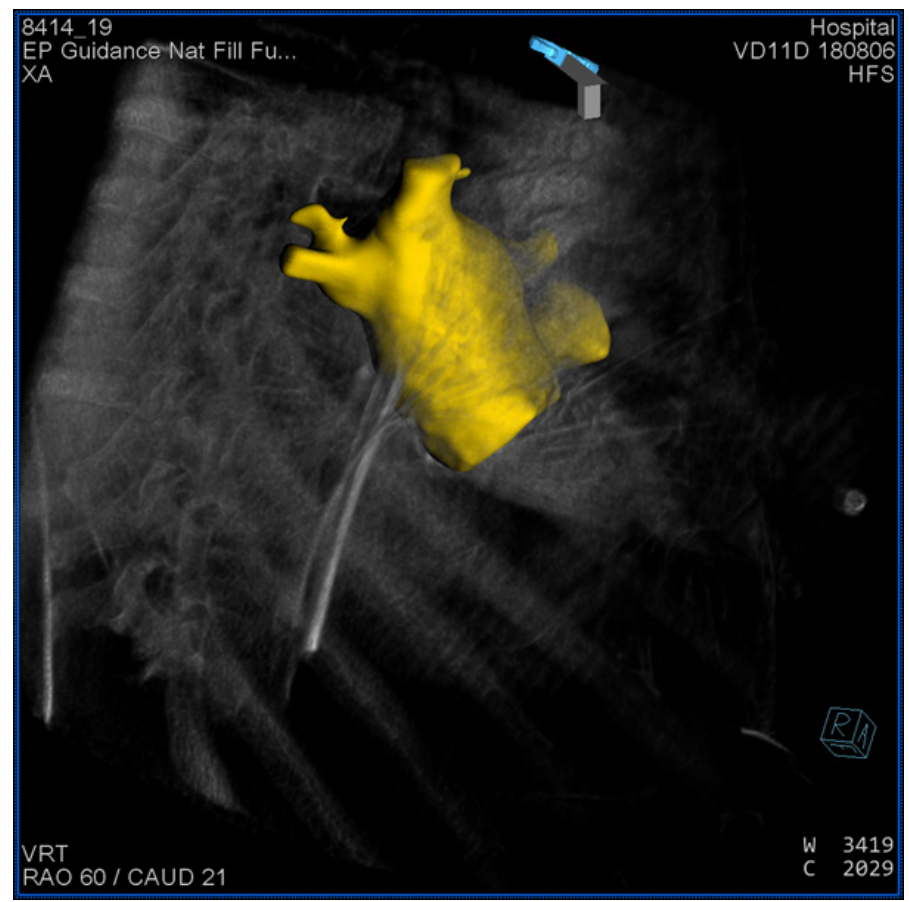

Figure2. 3D rotational angiography image of left atrium.

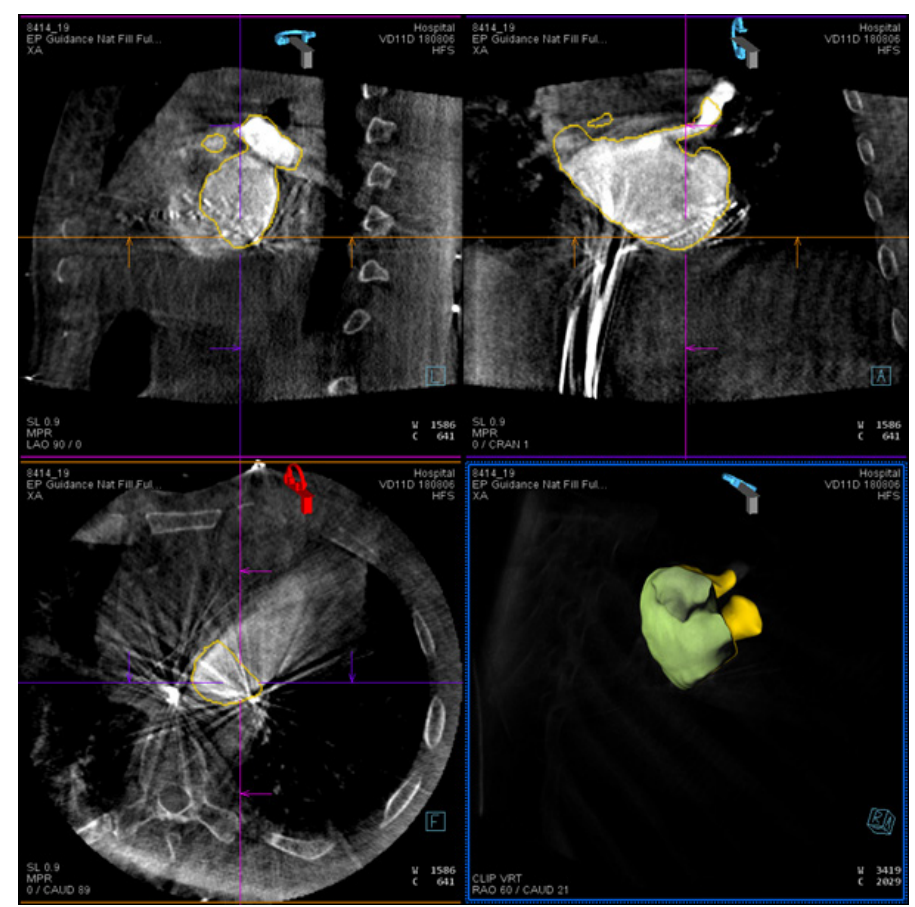

Figure3. 3D reconstruction of the left atrium

software used for rotational angiography was Siemens Artis Q. Automatic 3D reconstruction with Leonardo Workstation CT. Merging was performed using CARTOUNIVU Module and specific landmarks. (Figure 4). Subsequently circular isolation

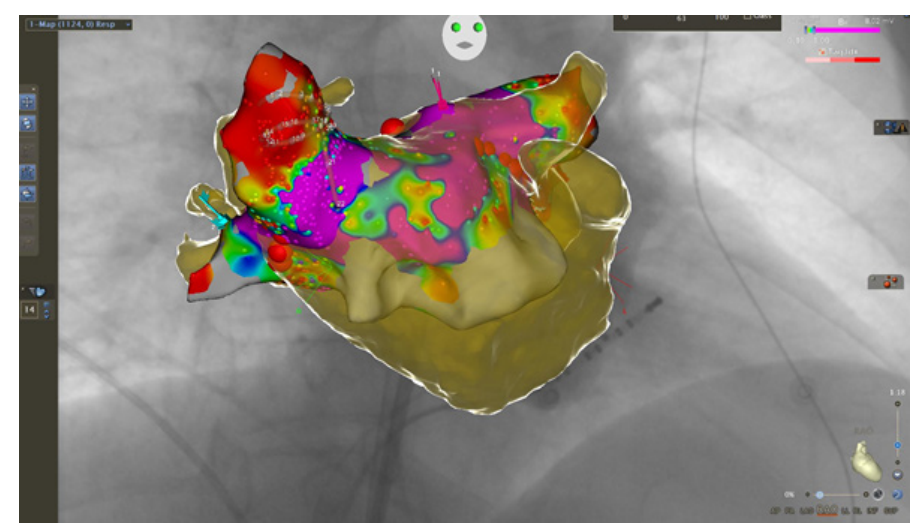

Figure4. 3DRA and EAM system merged - RAO $30^{\circ}$ view.

with circumferential point-by-point radiofrequency of all 4 pulmonary veins (ipsilateral with one circle) was performed. $12000 \mathrm{IU}$ of heparin was administered during the procedure with periodical activated clotting time (ACT) control.

Total procedure time was 2:40 h with fluoroscopy time 5:24 min (total radiation dose: $192.3 \mathrm{mGy}$, 3DRA dose: $177 \mathrm{mGy}$ ). Total number of RF applications was 60 with summarized application time of 32:18 $\mathrm{min}$.

After the procedure sinus rhythm was maintained and the patient was transferred to the intensive care unit, where after standard postprocedural echocardiography showed no effusion in the pericardial sac anticoagulation with dabigatran was administered. 
Due to lack of complications 3 days after the procedure the patient was discharged from the hospital in general good condition, without registered AF episodes.

\section{Discussion}

This case report provides data regarding the feasibility and benefits from the combination of preprocedural rotational angiography with electro-anatomical mapping. Due to the anatomical complexity and variation of the left atrium PVI remains one of the most challenging procedures in the electrophysiological range; therefore additional information in the form of higher quality images is in high demand.

Three-dimensional rotational angiography (3DRA) as an innovative method that allows near real-time 3D precise cardiac structure reconstruction during an ongoing CA procedure, and therefore can be used instead of conventional preprocedural CT or MRI or as a valuable addition to standard fluoroscopy ${ }^{(3)}$. It is generally agreed that fluoroscopy should be used as low as reasonably achievable (ALARA) ${ }^{(4)}$ in order to minimize the risk of adverse events, i.e. skin changes and malignancy, and in the case of 3DRA radiation exposure is lower than generally accepted values for cardiac interventional procedures ${ }^{(2)}$. Going further, limiting radiation exposure also concerns electrophysiologists, and can be achieved by initializing the $\mathrm{C}$-arm run from the control room. 3DRA appears to be a superior imaging method compared to CT and MRI due to avoiding volume mismatch connected with volume status, rhythm and chest geometry influenced by table geometry. However, high quality imaging is not the only benefit. 3DRA is advantageous in terms of logistics and cost-effectiveness when compared with CT and $\mathrm{MRI}^{(2,5)}$. Performing 3DRA in the electrophysiology laboratory allows logistic independence from other departments. The whole process of 3DRA takes place during the procedure, which is much easier to organize than scheduling a separate examination involving additional personnel and devices. Performing 3DRA requires an additional $14.4 \pm 3.2$ minutes, which is acceptable when taking into account better imaging ${ }^{(6)}$. Even though 3DRA is connected with additional fluoroscopy use because of its mechanism, it is highly profitable in reducing later fluoroscopy use and therefore consistent with the ALARA rule.

Since EAM together with the contact force sensing ablation catheter has been shown to reduce fluoroscopic exposure with a good effect on procedure time and success rate, it has become an inseparable part of electrophysiological procedures, including PVI. Moreover, it provides a wealth of information regarding arrhythmia and cardiac geometry and allows catheters to be manipulated without harmful fluoroscopy use ${ }^{(7)}$.

Combination of 3DRA and EAM brings even more benefits in terms of left atrial reconstruction and reduced radiation exposure. Even despite the additional 10 min needed to perform 3DRA it is profitable when considering procedure time, because of the improved image quality and easier catheter manipulation during the later part of $\mathrm{CA}^{(8)}$. PVI is known to be one of the most challenging ablation procedures; therefore safety and efficacy are of the highest priority, and can be achieved by using new technologies, i.e. 3DRA and EAM merger ${ }^{(9,10)}$.

Even though some experienced centers refuse to use 3DRA due to their ability to orientate in 2D fluoroscopy, a multicenter registry shows that image integration is highly beneficial regarding freedom from $\mathrm{AF}^{(11)}$. However, one should also remember about the very limited, yet present risk of an anaphylactic reaction connected with contrast administration.

As shown in our case and previous studies, 3DRA is a feasible and valuable tool during PVI procedures, which is expected to gain popularity among electrophysiologists. Because of its undeniable profits it facilities the CA procedure, and therefore helps to minimize dangerous $\mathrm{AF}$ complications. 3DRA is an effective method that has a role in the general aim to lower the worldwide cardiovascular burden.

\section{References}

1. Kirchhof P, Benussi S, Kotecha D, Ahlsson A, Atar D, Casadei B, et al. 2016 ESC Guidelines for the management of atrial fibrillation developed in collaboration with EACTS. Europace. 2016;18(11):1609-78.

2. De Potter T, Jr., Bardhaj G, Viggiano A, Morrice K, Geelen $P$. Three-dimensional rotational angiography as a periprocedural imaging tool in atrial fibrillation ablation. Arrhythm Electrophysiol Rev. 2014;3(3):173-6.

3. Orlov MV, Hoffmeister P, Chaudhry GM, Almasry I, Gijsbers GH, Swack T, et al. Three-dimensional rotational angiography of the left atrium and esophagus - A virtual computed tomography scan in the electrophysiology lab? Heart Rhythm. 2007;4(1):37-43.

4. Limacher MC, Douglas PS, Germano G, Laskey WK, Lindsay BD, McKetty MH, et al. ACC expert consensus document. Radiation safety in the practice of cardiology. American College of Cardiology. J Am Coll Cardiol. 1998;31(4):892-913.

5. Kriatselis C, Nedios S, Akrivakis S, Tang M, Roser M, Gerds-Li JH, et al. Intraprocedural imaging of left atrium and pulmonary veins: a comparison study between rotational angiography and cardiac computed tomography. Pacing Clin Electrophysiol. 2011;34(3):315-22.

6. Li JH, Haim M, Movassaghi B, Mendel JB, Chaudhry GM, Haffajee CI, et al. Segmentation and registration of three-dimensional rotational angiogram on live fluoroscopy to guide atrial fibrillation ablation: a new online imaging tool. Heart Rhythm. 2009;6(2):231-7.

7. Lee G, Hunter RJ, Lovell MJ, Finlay M, Ullah W, Baker $\mathrm{V}$, et al. Use of a contact force-sensing ablation catheter with advanced catheter location significantly reduces fluoroscopy time and radiation dose in catheter abla- 
tion of atrial fibrillation. Europace. 2016;18(2):211-8.

8. Knecht S, Wright M, Akrivakis S, Nault I, Matsuo S, Chaudhry GM, et al. Prospective randomized comparison between the conventional electroanatomical system and three-dimensional rotational angiography during catheter ablation for atrial fibrillation. Heart Rhythm. 2010;7(4):459-65.

9. Carpen M, Matkins J, Syros G, Gorev MV, Alikhani $\mathrm{Z}$, Wylie JV, et al. First experience of 3D rotational angiography fusion with NavX electroanatomical mapping to guide catheter ablation of atrial fibrillation. Heart Rhythm. 2013;10(3):422-7.

10. Nolker G, Asbach S, Gutleben KJ, Rittger H, Ritscher $\mathrm{G}$, Brachmann J, et al. Image-integration of intraprocedural rotational angiography-based 3D reconstructions of left atrium and pulmonary veins into electroanatomical mapping: accuracy of a novel modality in atrial fibrillation ablation. J Cardiovasc Electrophysiol. 2010;21(3):278-83.

11. Bertaglia E, Bella PD, Tondo C, Proclemer A, Bottoni $\mathrm{N}$, De Ponti R, et al. Image integration increases efficacy of paroxysmal atrial fibrillation catheter ablation: results from the CartoMerge Italian Registry. Europace. 2009;11(8):1004-10. 